\title{
Generación de valor con enfoque en la filosofía esbelta como facilitador en la percepción de valor al cliente (Value generation with focus on lean philosophy as a facilitator for customer perceived value)
}

\section{Marco Segura-Vargas José Nicolás Barragán Codina*}

\begin{abstract}
In the past twenty years, companies of different types of industry have focused their attention and resources trying to create more value for their customers, they have established according to their own judgment or consulting customers assuming they know exactly what they want and how they want it. However, the reality might be different. The lean philosophy defines the principles that any process (production or service) must follow to deliver more value and generate less waste. So this document proposes that if companies try to focus their efforts on delivering more value to their customers from the Lean perspective, they can generate a greater perception of value, therefore, raise their confidence and gain their loyalty. If it is practiced consistently over time they may achieve sustainable profitability.
\end{abstract}

Key words: customer value, lean philosophy, lean thinking, loyalty perceived value, profitability

JEL: M30, L15, L80, 014, O40

\footnotetext{
- Universidad Autónoma de Nuevo León, Facultad de Contaduría Pública y Administración, Centro de Desarrollo Empresarial y Posgrado, Av. Universidad S/N, San Nicolás de los Garza, N.L., México, C.P. 66455, (+52) 8113 4044 30, marco.segura@gmail.com

* Universidad Autónoma de Nuevo León, Facultad de Contaduría Pública y Administración, Centro de Desarrollo Empresarial y Posgrado, Av. Universidad S/N, San Nicolás de los Garza, N.L., México, C.P. 66455, (+52) 8113 4044 30, jose.barraganc@uanl.mx
} 
Resumen. En los últimos veinte años, compañías de distintos tipos de industria han enfocado su atención y recursos en crear más valor a sus clientes, y lo han establecido ya sea de acuerdo con su propio criterio, 0 al consultar a sus clientes y asumir que ellos saben qué es lo que quieren y cómo lo quieren. Sin embargo, la realidad podría ser un tanto distinta. Por su parte, la filosofía esbelta define los principios que cualquier proceso (de producción o servicio) debe seguir para entregar más valor y generar menos desperdicio. Entonces este documento propone que, si las compañías tratan de enfocar sus esfuerzos en entregar más valor a sus clientes desde la perspectiva Esbelta, podrán generar una mayor percepción de valor, por tanto, elevarán su confianza y obtendrán su lealtad. Si lo practican de forma sistemática con el paso del tiempo podrán lograr una rentabilidad sostenible del negocio.

Palabras clave: filosofía esbelta, lealtad, pensamiento esbelto, percepción de valor, rentabilidad, valor al cliente

\section{Introducción}

En la actualidad las compañías están en busca de hacer un uso más eficiente de los recursos y a la vez poder brindar más valor a sus clientes. De manera común se traduce en la práctica de las organizaciones como el conseguir ahorros significativos en la operación e intentar no sacrificar la calidad en el producto o servicio, pero también se suele interpretar como tratar de brindar valor agregado al cliente con un mismo o menor gasto operativo, o una mezcla de ambas ideas.

El concepto de valor al cliente es amplio y las compañías suelen perderse en sus intentos de brindar más valor al cliente, de tal manera que es posible que agreguen valor en algo que no tiene una importancia real para el cliente mismo. Esto es debido a que las compañías no precisan de conocer a bien los factores que influyen en la percepción de valor al cliente y cómo estos pueden potencializarse.

Al comprender la cadena de Calidad y Rentabilidad se conoce la importancia de la Percepción de Valor al cliente y su influencia en la rentabilidad de las empresas; esto es apoyado a su vez por la teoría de la Ventaja Competitiva la cual persigue enfocar los recursos siempre en favor del cliente. De tal forma, que al comprender el Valor enfocado al cliente y al conocer la conformación de la Percepción de Valor permite entonces 
combinarlo con los conceptos de la Filosofía Esbelta utilizados principalmente en la manufactura.

Este estudio realiza una propuesta al tratar de destacar la importancia y la valía que pueden obtener las compañías si enfocan sus esfuerzos actuales en la generación de valor al combinarlo con la filosofía Esbelta; de tal manera que puedan potencializar, hacer un uso eficiente y enfocar los recursos en acciones que incrementen la Percepción de Valor al Cliente.

\section{Objetivo, hipótesis y metodología de la investigación}

Se tiene por objetivo sustentar de manera teórica que la Percepción de Valor al Cliente se potencializa al aplicar los conceptos de la filosofía esbelta en cada uno de los factores que influyen en esta.

De tal forma, la hipótesis propuesta es que los factores que componen la Percepción de Valor al Cliente: Valor Funcional, Valor Social, Valor Emocional y el Precio, cuando son expuestos a los conceptos de la filosofía Esbelta incrementan la generación de valor al cliente. Dichos factores forman parte de los modelos teóricos que se describen posteriormente.

La metodología utilizada en esta investigación tiene un diseño no experimental dado que no hay alguna injerencia en las variables de estudio. El tipo de la investigación es exploratoria y descriptiva, por tanto, se requiere revisar las teorías que fundamentan la importancia de la generación de valor con un enfoque en la rentabilidad y competitividad. Además de comprender la teoría de la Percepción de Valor al Cliente y entender los conceptos fundamentales de la filosofía Esbelta. Por tanto, en el estudio se realiza una recolección de la información bibliográfica de las teorías señaladas con enfoques en sistemas de calidad, procesos y servicio. Para proseguir con la interpretación de la información recopilada, y obtener como resultado los factores que infieren en la Percepción de Valor al Cliente, además de identificar los estudios con objetivos similares a este. De tal manera, que al final se realice una reflexión de cómo generar mayor valor al utilizar estos conceptos. 


\section{Revisión de literatura}

La importancia de la generación de valor y cómo influye en la Percepción de Valor al cliente se describe en las siguientes teorías.

\section{Motor de rentabilidad}

Existen diversos estudios de análisis donde se demuestra la relación positiva entre la calidad del servicio, la calidad del producto y el costo con la percepción de valor al cliente, que a su vez tiene relación positiva con la lealtad del cliente (Parasuraman \& Grewal, 2000). Es claro que si el objeto intercambiado es un servicio y no un producto a mayor calidad de servicio se percibe como mayor valor por el cliente.

Para Parasuraman la percepción de valor es el elemento determinante para lograr la lealtad del cliente, otros autores como Heskett et al. (1994) han considerado a la satisfacción del cliente como un paso intermedio entre la percepción de valor y la lealtad del cliente, como se muestra en la Figura 1. Debido a que el cliente recompensa la satisfacción recurrente con su lealtad al paso del tiempo.

Figura 1: Motor de la rentabilidad

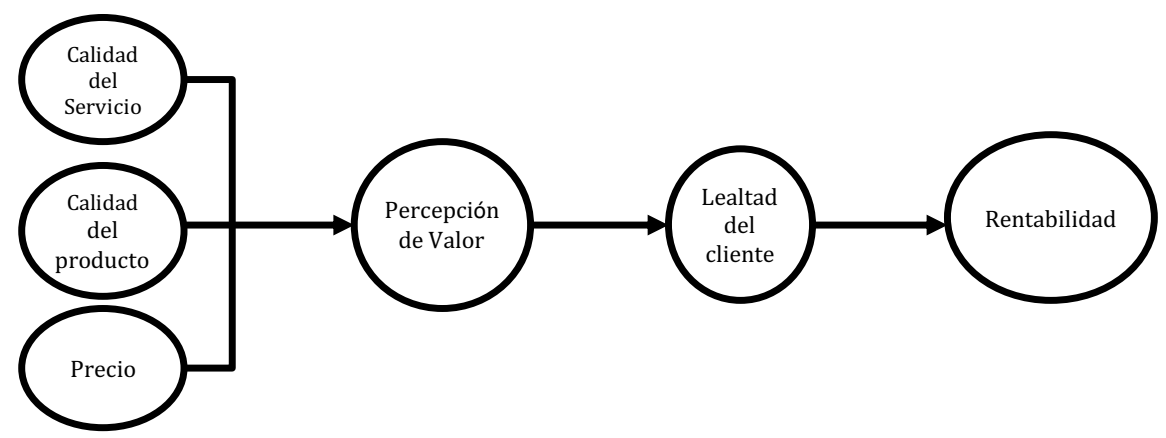

Fuente: Parasuraman \& Grewal (2000); Heskett, Jones, Loveman, Sasser, \& Shlesinger (1994)

Por consiguiente, cuando una compañía aumenta el valor al cliente genera un aumento en la percepción de valor que se refleja en la lealtad del mismo cliente, esta lealtad es un activo de la compañía que el cliente le 
genera a la compañía misma, esta es una relación ganar-ganar entre la compañía y el cliente, aun así, es poco frecuente observar que las compañías conozcan el verdadero valor de un cliente leal, además de no registrarlo en sus libros contables como un activo. Independiente de la industria mientras más permanece un cliente mayor es la rentabilidad a nivel general de la compañía (Reichheld \& Sasser, 1990). Los clientes con el paso del tiempo se vuelven más rentables al incrementar su frecuencia de compra, reducen los costos operativos, influyen en forma positiva en las compras de sus referidos $u$ otros clientes, esto incrementa el consumo de productos o servicios e incluso los de mayor valor.

\section{Ventaja competitiva}

En la actualidad las organizaciones dentro de un ambiente competitivo son exigidas a ser cada vez más eficientes desde el punto de vista financiero, y a ofrecer mejores productos y servicios a precios justos. Para esto las compañías de forma general optan por optimizar sus costos, aumentar su eficiencia operativa, sacrificar el margen de ganancia al bajar los precios (Porter M. , 1985) u ofrecer ofertas, introducir nuevos productos al mercado, o agregar nuevos servicios con la finalidad de estar a la par de sus competidores.

Algunas compañías optan por ofrecer y entregar más valor al cliente con la finalidad de ser más competitivas y poder diferenciarse de su competencia (Womack \& Jones, 1996); de esta manera llaman la atención del cliente, ganan mercado y crece la rentabilidad del negocio. Por lo tanto, es común encontrarlo incluido en la planeación estratégica de las compañías de las diferentes industrias de bienes y servicios (Porter M. , 1998).

El valor agregado no se trata de una característica más del producto 0 el servicio, sino es una serie de pequeñas unidades de valor que se le agregan en todo aquello que toca al cliente a lo largo de la cadena de servicio. A medida que se agregan unidades de valor en beneficio para el cliente se construye una cadena de valor. Por lo que las compañías que enfocan sus esfuerzos en generar una mejor cadena de valor a sus clientes incrementan su ventaja competitiva. (Heskett, Jones, Loveman, Sasser, \& Shlesinger, 1994; Levitt, 1960; Woodruff, 1997) 
De una u otra manera, las compañías también mantienen una cadena de servicio, al final siempre existe un cliente interno o externo a quien se le brinda un servicio independiente de la industria o giro. Por lo que la competitividad se construye desde dentro hacia afuera de las organizaciones.

Cuando la cadena de valor se realiza de manera sistemática y al anteponer siempre de manera genuina el interés del cliente, las compañías son retribuidas con la confianza del consumidor y a lo largo del tiempo se convierte en lealtad (Parasuraman \& Grewal, 2000). De tal manera que las compañías pueden optar por la generación de valor como una ventaja competitiva y de diferenciación con la competencia.

\section{Valor}

Para entender de mejor manera el concepto de valor es importante conocer las diferencias cuando se refiere a "valor del" área de interés y "valor al" área de interés. El área o áreas de interés se define como la entidad o entidades que tienen relación directa o indirecta con una organización, como pueden ser clientes, proveedores, empleados, accionistas, comunidad, entre otros. (Conocido en inglés como "Stakeholder"). El Valor del área de interés se le conoce a aquellos beneficios que el área de interés brinda a la organización. Por ejemplo, en el caso de los clientes, pueden tener la capacidad de atraer a nuevos y mejores clientes (Valenzuela, Garcia de la Madariega, \& Blasco, 2007), los proveedores pueden coadyuvar a la organización en compartir recursos o evitar retrabajos, los empleados pueden ser más eficientes 0 participar de manera activa en iniciativas de mejora e innovación, los accionistas inyectan recursos para financiar proyectos, entre otros. Adicional, no solo se deben contabilizar los beneficios en el valor actual sino también en su potencial a futuro.

El valor al área de interés es similar aunque conceptualizado desde la perspectiva del área de interés misma, es decir, se refiere a todas las unidades de valor que recibe el área de interés por una o varias compañías u organizaciones; el valor entregado se refiere a todo el valor que el área de interés recibe durante el proceso que le llevó a resolver su requerimiento 0 problemática, desde que tiene la necesidad de satisfacer el requerimiento 0 
solucionar su problema hasta que queda entregado o resuelto a su entera satisfacción, y en algunas casos hasta el final de su vida útil.

Percepción de valor

La percepción de valor está definida como la relación entre el valor agregado y el desperdicio generado al recibir un bien, o un servicio. Desde el punto de vista del cliente, de acuerdo con Zeithaml (1988) el cliente entiende cuatro distintas definiciones de valor, cada una de ellas son válidas y dependen del contexto que se encuentra el cliente: (1) valor es precio bajo, (2) valor es lo que quiero que sea de un producto, (3) es el valor que consigo por el precio que pago, (4) valor es lo que consigo por lo que doy.

A partir de finales de los años ochenta comenzaron a surgir estudios relacionados con la percepción de valor y los factores que influyen en este. $A$ manera de consenso por los diferentes autores los factores o variables fueron englobados en dimensiones.

Sheth et al. (1991) consideró cinco rubros que influían en la percepción de valor que llamó valores, el valor social, el emocional, el funcional, epistémico y emocional. Groh (1995) le llamó factores de influencia en la percepción de valor y distinguía al cognitivo, el psicológico, el interno y externo. Grönroos (1997) solo considera dos factores el factor cognitivo y el factor emocional o psicológico.

Sweeney y Soutar (2001) utilizaron 3 dimensiones que identifican la percepción del cliente, el valor funcional, el valor social y el valor emocional. En la dimensión funcional incluyeron factores como el precio, calidad y versatilidad, entendida como la capacidad del producto de adaptarse a un uso práctico.

Por su parte, Sanchez et al. (2006) desarrollaron 24 rubros agrupados en 2 dimensiones. En la dimensión de la funcionalidad se consideran aspectos como la evaluación del establecimiento, el trato personal, la calidad y el precio. La dimensión afectiva se divide en dos, el rubro emocional (relacionado a los sentimientos y emociones) y el rubro social (relacionado con el impacto social de la compra realizada.

Fandos Roig et al. (2006) consideraron seis valores que influyen de forma directa en la percepción de valor, los cuales son: emocional, social, funcional, personal, servicio recibido y precio. Smith y Colgate (2007) 
desarrollaron cuatro valores para determinar la percepción de valor, el funcional o instrumental, experiencia, simbólico o expresivo y costo/sacrificio.

De tal manera, los estudios relacionados con la Percepción de Valor se concentran en el resultado de la evaluación final que realiza el cliente en diferentes aspectos o dimensiones, pero dejan de lado una visión más global de la generación de valor como un sistema que de manera continua influye en la Percepción de Valor, y es dónde la Filosofía Esbelta realiza un aporte en este sentido.

\section{Filosofía esbelta}

Los sistemas de calidad en Japón se popularizaron después de las 2a Guerra mundial a raíz de la fuerte depresión económica que vivía la nación donde las compañías para subsistir optaron por diferentes medidas de eficiencia para reducir costos y optimizar procesos productivos. Para esto las compañías japonesas trataron de igualar los sistemas de producción utilizados en los Estados Unidos que comenzaron y evolucionaron con el sistema de producción del modelo-T por Ford de los años veinte. A diferencia de los sistemas de producción norteamericana, en las industrias japonesas los recursos económicos eran limitados, el mercado era menor y estaba deprimido y no contaban con una red de proveedores semejante a la de Estados Unidos.

Por su parte, las compañías automotrices japonesas adaptaron el sistema de producción en serie de Ford, sin embargo, necesitaban conseguir que fuera de alta calidad, de bajo costo, con tiempos cortos en los cambios de producción, y además ser más flexible.

De esta manera surgió el Sistema de Producción Toyota (por sus siglas en inglés: TPS) desarrollado por Taiichi Ohno (Liker, 2004) director general en la fábrica de automóviles Toyota entre 1948 y 1975 en Japón. Dicho sistema es más que la utilización de herramientas de mejora continua, es un mecanismo de operatividad y gestión de toda una organización basada en cuatro rubros:

- Filosofía: las decisiones son tomadas a largo plazo, a expensas de lo que suceda a corto plazo 
- Proceso: busca el flujo continuo y elimina el desperdicio (muda, del japonés)

- Gente y Socios: Siempre tiene un profundo respeto por la gente, desde clientes, empleados, proveedores. Además de fomentar el trabajo en equipo.

- Resolución de los problemas: Fomenta de forma consistente y sistemática la mejora. Procura en cada momento la reflexión y el aprendizaje.

Este sistema fue la respuesta de Ohno para hacer de Toyota una compañía productiva y rentable a lo largo del tiempo, y así superar las adversidades de su tiempo.

Los sistemas de calidad utilizados en Japón fueron entendidos por el resto del mundo como la utilización de herramientas estadísticas para encontrar áreas de oportunidad o problemas y resolverlos en un ciclo continuo que llamaron "círculos de calidad". Estas herramientas de mejora fueron aplicadas en muy diversos tipos de industrias en diferentes países. Sin embargo, con el paso del tiempo estos esquemas de mejora no fueron tan exitosos y contundentes como lo aplicado en la empresa Toyota en Japón.

Womack \& Jones $(1996 ; 2005)$ al hacer un ejercicio de observación con base a sus vivencias en una fábrica de Toyota descubrieron que el verdadero éxito de Toyota es la utilización del TPS. En 1996 escribieron y dieron a conocer lo que ahora se conoce como la filosofía Esbelta (en inglés, al comienzo llamada Lean Manufacturing, posteriormente Lean philosophy), en español fue traducido y popularizado como Manufactura Esbelta. Hoy en día es utilizada en la industria de la manufactura en todo el mundo.

La aportación de Womack y Jones fue obtener la esencia del TPS como un pensamiento de transformación para las organizaciones con la finalidad de hacerlas más eficientes, productivas, con alto grado de compromiso y un gran sentido de colaboración de los empleados y proveedores.

El pensamiento Esbelto como lo llamaron sus autores es considerado como la filosofía del mejoramiento de los procesos de manufactura y servicios (Bhasin \& Burcher, 2006), se basa en cinco principios: dar valor al cliente, eliminar desperdicios y actividades no generadoras de valor, procurar siempre el flujo continuo, realizar solo actividades o tareas solicitadas por el 
cliente y buscar la perfección mediante la mejora continua (Womack \& Jones, 1996).

Como se observa en la Figura 2, la filosofía Esbelta tiene el objeto de aumentar la creación de valor para incrementar la percepción de valor al cliente. El principal "mensaje" detrás de los cinco principios es cuando una organización interpreta el valor que desea otorgar al cliente bajo determinados términos, el valor estará fluyendo por toda la cadena de valor hasta ser "arrastrado" por el cliente en el momento y cantidad deseada.

Figura 2: Esquema de la filosofía esbelta

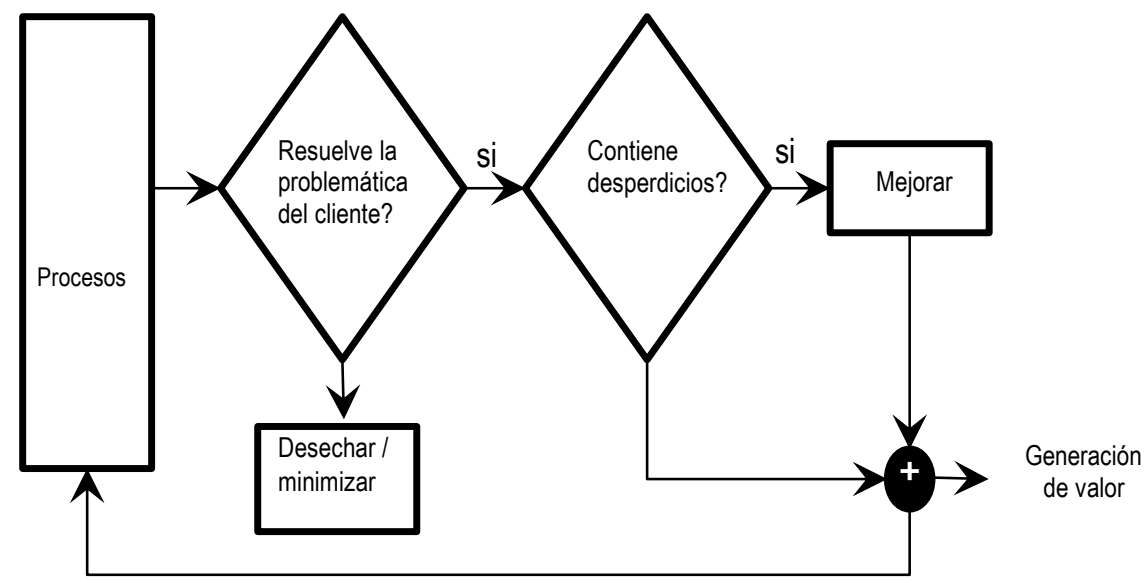

Fuente: Elaboración propia

La implementación de la filosofía Esbelta dentro de las organizaciones no es tarea sencilla, algunas compañías se han quedado en el intento al utilizar solo las herramientas de mejora a diferencia de lo desarrollado por Toyota, para ser exitosa debe formar parte de la cultura de la organización. También hay casos de éxito donde las compañías han utilizado y adaptado esta filosofía a su propio entorno y bajo su propio entendimiento, al no tratarse de una guía de mejores prácticas, cada compañía es "Esbelta" bajo su propia manera de vivir cada principio. La clave de la implementación es el convencimiento de la alta dirección para después llegar a todos los niveles de la organización. 
Las investigaciones han mostrado que la industria del servicio se comporta de cierta manera similar a la manufactura (Zeithaml, 1988) aun cuando ambas industrias pudieran parecer muy distintas, en donde el costo y la calidad tienen una relación directa con el servicio prestado. De forma tal, los principios de la filosofía Esbelta pueden aplicarse de la misma manera en el servicio (Teehan \& Tucker, 2010; Womack \& Jones, 2005), primero se clarifica el concepto de valor al cliente, y después se busca que todo el servicio tenga un flujo continuo siempre a partir de la demanda del cliente, además de comprometerse en la perfección mediante la mejora continua.

En diferentes partes del mundo existen casos de éxito de compañías de servicio que han utilizado la filosofía Esbelta como un sistema de trabajo para concentrarse en las necesidades del cliente y lo que es importante para él, compañías de salud y operación en oficinas han tenido mayor éxito en adoptar esta filosofía, aunque en México existen limitados casos documentados de implementación Esbelta en esta industria.

\section{Resultados}

Esta investigación teórica y documental sirvió para mostrar como los estudios de la Percepción de Valor han coincidido en mayor medida en los constructos que la conforman, aun cuando han sido estudiados en diferentes industrias, como la banca, la hotelera, el turismo, la industrial y otras (Heinonen, 2004; Sanchez, Callarisa, Rodríguez, \& Moliner, 2006; Fandos, 2006; Cengiz \& Kirkbir, 2007; Pihlström \& Brush, 2008). La Tabla 1 muestra el resumen de las variables encontradas en la literatura y las variables propuestas en el estudio. De acuerdo con lo anterior y las teorías analizadas se realizan las siguientes definiciones conceptuales para las variables propuestas en la investigación: Valor Funcional, Precio, Valor Emocional y Valor Social.

El Valor Funcional se compone de la calidad del producto y la calidad del servicio. La calidad del producto es definida como el éxito de un producto en superar las expectativas del cliente; y de acuerdo con Garvin (1987) existen ocho dimensiones como: el desempeño, las características secundarias, la confiabilidad, la conformidad, la durabilidad, la utilidad, la estética y la calidad percibida. Por su parte, la calidad del servicio se refiere al juicio que realiza el cliente por la superioridad del servicio recibido basado en sus expectativas (Zeithaml, 1988). De acuerdo con la herramienta 
SERVQUAL (Calidad de Servicio) desarrollado por Parasuraman (1988) consta de cinco elementos: los elementos tangibles, la fiabilidad, la capacidad de respuesta, la seguridad y la empatía.

Tabla 1. Resumen de la literatura y las variables de estudio

\begin{tabular}{|c|c|c|c|c|c|}
\hline Autor & Percepción del valor & $\begin{array}{c}\text { Valor } \\
\text { Funcional }\end{array}$ & Precio & $\begin{array}{c}\text { Valor } \\
\text { Emocional }\end{array}$ & $\begin{array}{l}\text { Valor } \\
\text { Social }\end{array}$ \\
\hline $\begin{array}{l}\text { Sheth et al. } \\
\text { (1991) }\end{array}$ & $\begin{array}{l}\text { Valor Social } \\
\text { Valor Emocional } \\
\text { Valor Funcional } \\
\text { Valor Epistémico } \\
\text { Valor condicional }\end{array}$ & $\checkmark$ & & $\checkmark$ & $\checkmark$ \\
\hline Groth (1995) & $\begin{array}{l}\text { Cognitivo: percepción de utilidad } \\
\text { Psicológico } \\
\text { Interno } \\
\text { Externo }\end{array}$ & $\checkmark$ & & $\begin{array}{l}\checkmark \\
\checkmark\end{array}$ & $\checkmark$ \\
\hline $\begin{array}{l}\text { Grönroos } \\
(1997)\end{array}$ & $\begin{array}{l}\text { Cognitiva } \\
\text { Emocional (psicológico) }\end{array}$ & $\checkmark$ & & $\checkmark$ & \\
\hline $\begin{array}{c}\text { Ruyter, J, \& } \\
\text { Peeters, (1997) }\end{array}$ & $\begin{array}{l}\text { Dimensión Emocional o valor intrínseco } \\
\text { Dimensión Funcional o valor extrínseco } \\
\text { Dimensión Lógica }\end{array}$ & $\checkmark$ & & $\checkmark$ & \\
\hline $\begin{array}{c}\text { Sweeney, } \\
\text { Soutar y } \\
\text { Johnson (1999) }\end{array}$ & $\begin{array}{l}\text { Valor Social (aceptabilidad) } \\
\text { Valor Emocional } \\
\text { Valor Funcional (precio/ valor por el dinero) } \\
\text { Valor Funcional (desempeño/ calidad) } \\
\text { Valor Funcional (versatilidad) }\end{array}$ & $\checkmark$ & $\checkmark$ & $\checkmark$ & $\checkmark$ \\
\hline $\begin{array}{l}\text { Sweeney \& } \\
\text { Soutar (2001) }\end{array}$ & $\begin{array}{l}\text { Dimensión Funcional (económica y calidad) } \\
\text { Dimensión Social } \\
\text { Dimensión Emocional }\end{array}$ & $\checkmark$ & $\checkmark$ & $\checkmark$ & $\checkmark$ \\
\hline $\begin{array}{l}\text { Sanchez et al. } \\
\qquad(2006)\end{array}$ & $\begin{array}{l}\text { Dimensión Funcional (instalaciones, calidad, } \\
\text { precio) } \\
\text { Dimensión Afectiva (emociones, social) }\end{array}$ & $\checkmark$ & $\checkmark$ & $\checkmark$ & \\
\hline $\begin{array}{l}\text { Heinonen } \\
(2004)\end{array}$ & $\begin{array}{l}\text { Dimensión Técnica (atributos básicos del } \\
\text { servicio) } \\
\text { Dimensión Funcional (atributos relacionados } \\
\text { con la entrega del servicio) } \\
\text { Dimensión Temporal (atributos del tiempo de } \\
\text { entrega) } \\
\text { Dimensión Espacial (atributos del lugar } \\
\text { donde se entrega el servicio) }\end{array}$ & $\checkmark$ & & & \\
\hline $\begin{array}{c}\text { Smith \& } \\
\text { Colgate }(2007)\end{array}$ & $\begin{array}{l}\text {-Valor funcional/ Instrumental } \\
\text {-Valor experiencia/hedonista } \\
\text {-Valor simbólico/expresivo } \\
\text {-Valor costo/sacrificio }\end{array}$ & $\begin{array}{l}\checkmark \\
\checkmark\end{array}$ & $\checkmark$ & $\checkmark$ & $\checkmark$ \\
\hline
\end{tabular}

Fuente: Elaboración propia 
El precio se define como el valor monetario que paga el cliente al proveedor para recibir un producto o un servicio, por otra parte, la percepción del precio desde la perspectiva del cliente se refiere a la evaluación que realiza el cliente sobre la erogación monetaria de todos los gastos incurridos antes, durante y después de haber adquirido el producto o servicio.

El Valor Emocional de acuerdo con Richins (1997) lo define como la evaluación afectiva y sentimental que el cliente genera en cada punto de contacto. El factor del Aspecto Emocional en el servicio está compuesto por dos elementos (Sørensen, 2008), la interna que es aquella en donde el consumidor tiene una emoción previa a recibir un servicio y la externa que se refiere a la reacción o el efecto después de recibir un servicio.

El Valor Social de acuerdo con Sheth at al (1991) se refiere al valor simbólico de identidad derivado de la posesión de un producto, o marca. Aunque también se puede incluir lo correspondiente a la imagen social y reputación que tiene la compañía que produce un bien o servicio (Callarisa, Moliner \& Sanchez-Garcia, 2011). Sánchez at al. (2006) en sus estudios enfocados en servicio hacen referencia a la variable social como la percepción de un estatus social o nivel de vida que se representa por la adquisición de cierto servicio.

Aun con lo anterior y después de la revisión de la literatura, se encuentra que ha sido poco estudiado la influencia de la Percepción de Valor al cliente en empresas que han implementado los conceptos de la Filosofía Esbelta. Posiblemente esto se deba a que usualmente las empresas generan actividades de mejora en el sentido de la empresa hacia el cliente y no del cliente a la empresa, y esto no necesariamente conlleva a un incremento en el valor que el cliente percibe.

\section{Análisis de resultados: Generación de valor con enfoque esbelto}

De acuerdo con la literatura, no todo el valor agregado impacta de manera positiva en la percepción de valor al cliente, esto significa que los esfuerzos que las compañías realizan en agregar valor a sus productos o servicios en ocasiones no son percibidos como un beneficio real para el cliente. Es decir, el cliente puede percibir el valor agregado, pero no quiere decir que se soluciona un problema de forma automática. Ahora bien, es factible potencializar y enfocar la generación de valor al adjuntar los conceptos de 
Valor, Percepción de Valor y la Filosofía Esbelta como se muestra en la Figura 3.

Figura 3: Generación de valor enfoque esbelto

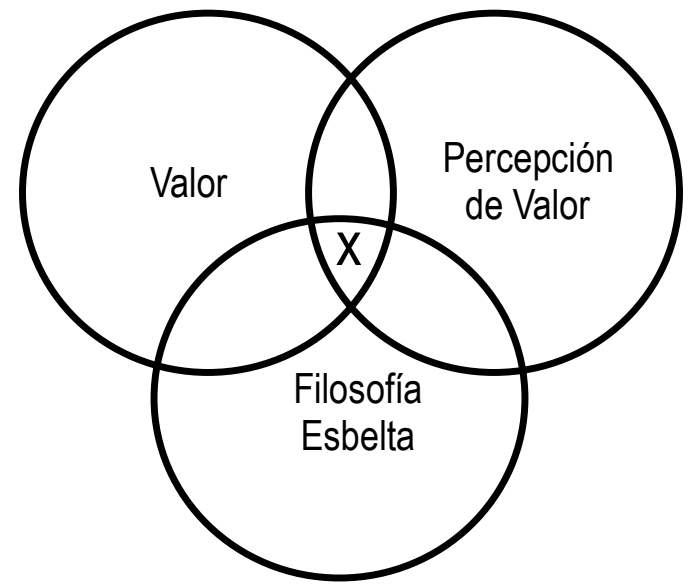

x: generación de valor

Fuente: Elaboración propia

De tal manera, la generación de valor al cliente con enfoque en la filosofía Esbelta tiene la finalidad de dirigir los esfuerzos de las compañías en rubros que brindan beneficio hacia el cliente en cada una de las dimensiones que influyen en la percepción de valor. Es decir, se potencializan los factores que influyen de forma directa en la percepción de valor, como se muestra en la Figura 4. Por tanto, las compañías obtienen beneficios adicionales al tener objetivos claros, enfoque en metas, ahorro en recursos, entre otros.

De tal manera, para poder lograr superar las expectativas del cliente sobre el producto desde la óptica de la filosofía Esbelta, se requiere conocer: qué es lo que el cliente está tratando de resolver con el producto, cómo el producto se está adecuando a las necesidades del cliente, identifica si el producto es entregado cómo y dónde el cliente lo requiere, además si la cantidad de producto es adecuada a su necesidad sin quedarse con excedentes. Para que el servicio sobrepase las expectativas del cliente se deberán enfocar los esfuerzos en: disminuir los tiempos de entrega, disminuir 0 evitar filas por parte del cliente, disminuir movimiento o traslados, evitar 
acciones repetitivas realizadas por el cliente, limitar el contacto cuando realmente se requiera, entre otros.

Figura 4: Dimensiones de la percepción de valor y la filosofía esbelta FILOSOFIA ESBELTA

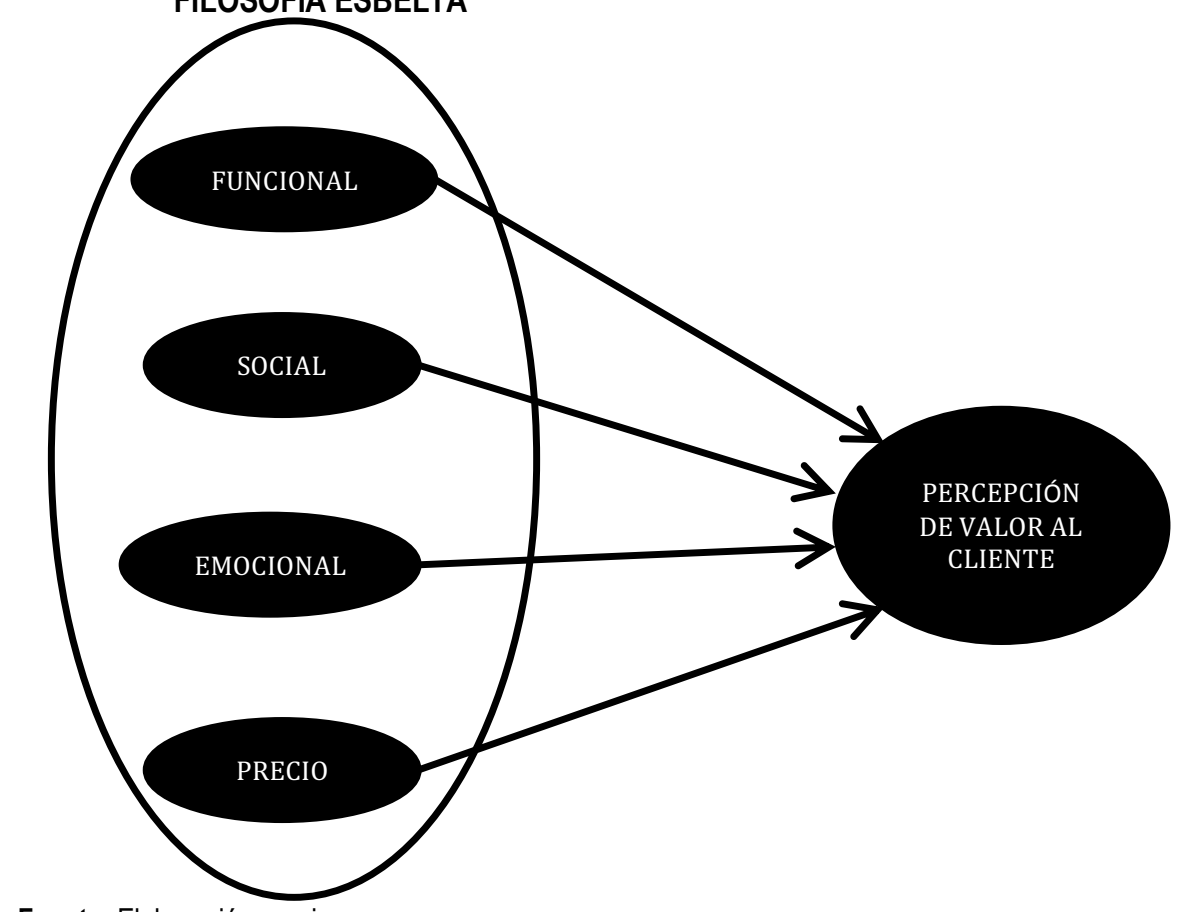

Fuente: Elaboración propia

Al tomar en cuenta los conceptos de la Filosofía Esbelta con perspectiva en la empresa, las mudas tienen una relación directa con el costo (Womack \& Jones, 1996), mientras más desperdicios se generen en el proceso que atiende o soluciona la problemática del cliente los costos se trasladan directamente al consumidor en un incremento en el precio. Ahora bien, desde la perspectiva del cliente, el consumidor se ve obligado a asumir los costos de las actividades que no le generan valor para conseguir su objetivo final, tal es el caso del costo de transportación y movimiento cuando no recibe el servicio en el momento y lugar deseado, el sobrecosto del producto cuando recibe más de lo solicitado, y el costo adicional generado 
cuando no se cumplen las especificaciones originales solicitadas por el cliente.

Para incrementar la percepción de valor en el factor emocional bajo la óptica de la filosofía esbelta, es necesario primero conocer todos los puntos de contacto entre el cliente y el proveedor, refiriéndose a todas las interacciones donde existe un acercamiento entre el cliente y empleados, instalaciones, publicidad, entre otros. Una vez identificados los puntos de contacto, se requerirá identificar aquellos que son generadores de valor y los que son muda. También se deberá identificar en dónde ocurren cuellos de botella que impiden el flujo continuo del proceso de servicio. Las acciones de mejora que se generen a partir de incrementar la satisfacción en los puntos generadores de valor, la eliminación de las mudas y los cuellos de botella generarán un incremento en la percepción de valor del cliente en el aspecto emocional.

Bajo la perspectiva Esbelta, para incrementar la percepción de valor en el factor social se deberá identificar aquello que el cliente busca en la marca, cómo ocurre y en qué momento se presenta esta identificación. De tal forma que deberán enfocarse los esfuerzos en incrementar, incentivar, transformar continua y positivamente estos momentos

Además, al buscar un enfoque siempre dirigido al cliente y no a la empresa, las compañías pueden adaptarse y flexibilizar su operación para cubrir y resolver las necesidades y problemas del cliente.

El reto para las compañías es hacerlo de tal manera que el flujo de valor sea continuo, eliminar de manera consistente los desperdicios no generadores de valor, hacerlo de una manera eficiente y de forma sistemática. Lo anterior no significa que se pueda dejar de poner atención en los aspectos comúnmente cubiertos y ampliamente estudiados para incrementar la satisfacción del cliente, al utilizar los conceptos de la filosofía esbelta fungen como un multiplicador al ser aplicados en los factores funcional, social, emocional y el precio.

Si bien, los conceptos de la Percepción de valor y la Filosofía Esbelta han sido ampliamente estudiados, la combinación de ellos como en el caso de esta propuesta han sido poco documentados, de tal manera que se abre la posibilidad de realizar investigaciones para conocer de forma profunda el grado de influencia y la aplicabilidad de los conceptos de la Filosofía esbelta 
en la Percepción de Valor en diferentes tipos de industrias. La Figura 5 muestra un modelo conceptual propuesto para el estudio del fenómeno.

Figura 5: Modelo conceptual de Percepción de Valor por medio de la Filosofía Esbelta

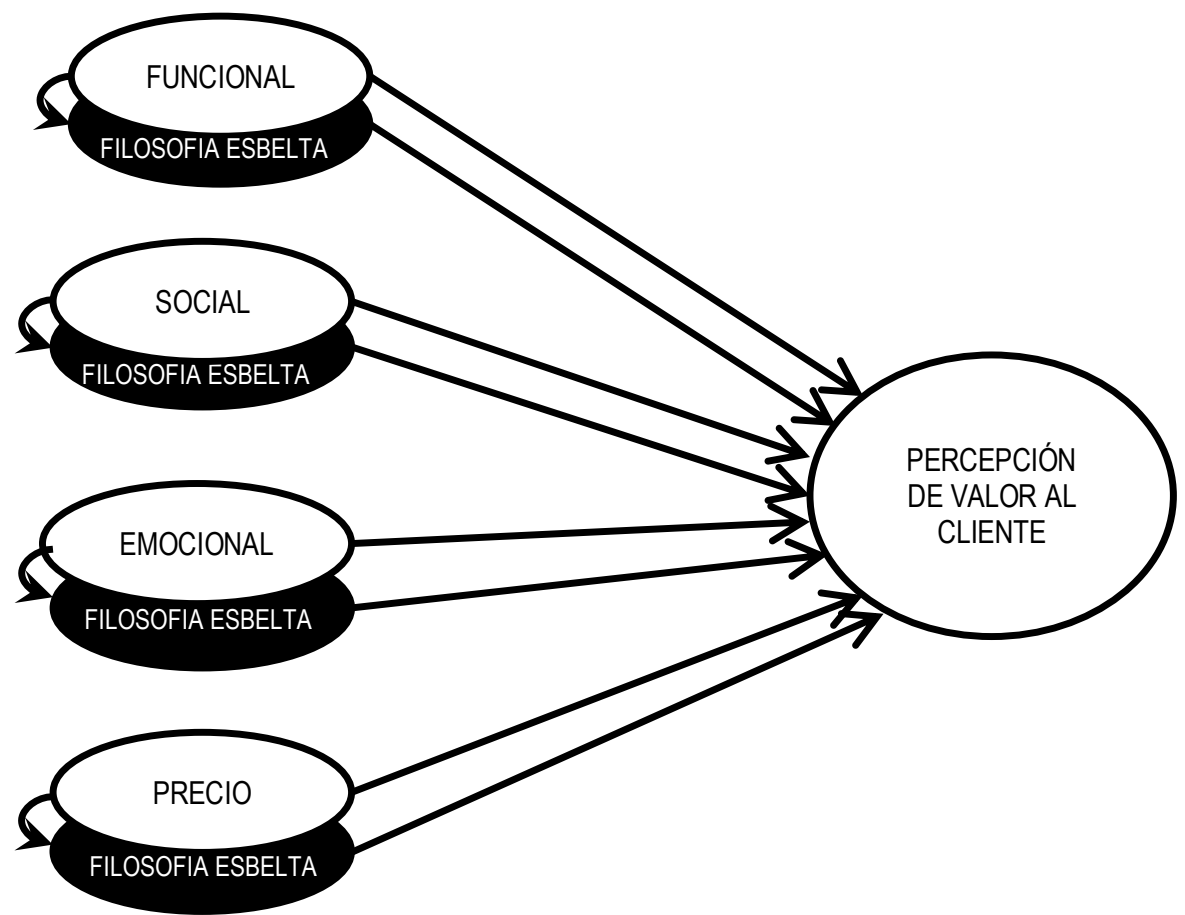

Fuente: Elaboración propia

\section{Discusión}

La creación de valor con enfoque en la filosofía Esbelta adquiere un sentido alterno al tradicional debido a que los esfuerzos se concentran en solucionar solo las problemáticas o requerimientos del cliente, por medio de un flujo continuo, y de forma consistente la eliminación de desperdicios. Si este proceder se conserva a lo largo de toda la cadena de valor, los productos o servicios tenderán siempre a mejorar desde el punto de vista del cliente. Esto genera confianza más rápido en el cliente que más tarde se traduce en 
lealtad a la empresa, y si se realiza de manera sostenible en el tiempo conduce a la rentabilidad de la misma.

$\mathrm{Al}$ introducir esta forma de crear valor en las diferentes dimensiones que conforman la percepción de valor del cliente potencializan los mismos y se enfocan de manera eficiente los recursos en entregar más y mejor valor a los clientes al menor costo. Porque al final se le entrega al cliente el producto y servicio que quiere, en la cantidad deseada, en el momento elegido y en el lugar donde el cliente lo prefiere. De tal manera que los clientes y las compañías se ven siempre beneficiadas al no haber desperdicios en tiempo, producto, inventario, y otros recursos. Por tanto, el valor se comparte y todos ganan.

Esta alternativa de generación de valor representa un cambio en la forma de cómo las compañías se organizan y se presentan a sus clientes, más allá de fungir como proveedores de productos o servicios, se convierten ahora en socios del cliente diseñados para resolver un determinado problema 0 atender un requerimiento. Es importante que las compañías de cualquier industria puedan comprender el grado de impacto que pueden llegar a generar al cliente y al negocio si consideran esta alternativa de generación de valor como uno de sus impulsores en su estrategia y desempeño diario.

Aunque este documento está enfocado al cliente como consumidor final y la compañía como el proveedor, podría también aplicar el mismo pensamiento teórico si se considera al "cliente" como un elemento de las áreas de interés, sería entonces factible poder aplicar los conceptos de la filosofía Esbelta como un facilitador en la generación de valor a los empleados, proveedores, accionistas, entre otros. Es decir, la compañía puede estar en constante búsqueda de generación de valor en todas las entidades que la conforman y así tener un beneficio completo.

\section{Referencias}

Bhasin, S. \& Burcher, P. (2006). Lean view as a philosophy, Journal of Manufacturing Technology Management, 17(1), 56-72.

Callarisa, L. J., Moliner, M. A. \& Sanchez-Garcia, J. (2011). Perceived value in industrial cluster: A multidimensional perspective, Journal of Business \& Industrial Marketing, 26(2), 132-145.

Cengiz, E., \& Kirkbir, F. (2007). Customer perceived value: The development of a multiple item scale in hospitals, Problems and Perspectives in Management, 5(3), 252-267. 
Fandos, J. C., Sanchez, J., Moliner, M. A. \& Llorens, J. (2006). Customer perceived value in banking services, International Journal of Bank Marketing, 24(5), 265-283.

Fandos, J. S. (2006). Customer Percived value in the banking services, International Journal of Bank Marketing, 24(5), 266-283.

Garvin, D. (1987). Competing on the eight dimensions of quality, Harvard Business Review, 65(6), 101-109.

Grönroos, C. (1997). Valur-driven relational marketing: from products to resources and competences, Journal of Marketing Management, 13(5), 407-420.

Groth, J. C. (1995). Important factor in the sale and pricing of services, Management Decision, 33(7), 29-34.

Grupo Bimbo México. (s.f.). Recuperado el 14 de Marzo de 2015, de http://www.grupobimbo.com/es/grupo-bimbo/mision-vision-y-valores.html

Heinonen, K. (2004). Time and location as customer perceived value drivers. Helsinki, Finlandia: Swedish School of Economics and Business Administration, Department of Marketing and Corporate Geography.

Heskett, J., Jones, T., Loveman, G., Sasser, W. \& Shlesinger, L. (1994). Putting the serviceprofit chain to work, Harvard Business Review, 86(7), 118-129.

Levitt, T. (1960). Marketing myopia, Harvard Business Review, 38(4), 24-47.

Liker, J. (2004). The Toyota way: 14 management principles from the world's greatest manufacturer. New York: McGraw-Hill.

McDonald's México. (s.f.). Recuperado el 14 de Marzo de 2015, de http://mcdonaldsprefeco.mex.t//704296_Mision-y-vision.html

Mision Southwest. (s.f.). Southwest. Recuperado el 14 de Marzo de 2015, de https://espanol.southwest.com/html/about-southwest/

Parasuraman, A., \& Grewal, D. (2000). The impact of technology on the quality-value-loyalty chain: A research agenda, Journal of the Academy of Marketing Science, 28(1), 168174.

Parasuraman, A., Zithalm, V. \& Berry, L. (1988). SERVQUAL: A multiple-item scale for measuring consumer perceptions of service quality, Journal of Retailing, 64(1), $12-40$.

Pihlström, M. \& Brush, G. (2008). Comparing the perceived value of information and entertainment mobile services, Phsycology and Marketing, 25(8), 732-755

Porter, M. (1985). Competitive advantage: Creating and sustaining superior performance. New York: The Free Press.

Porter, M. (1998). Stop imitating, and get to the real strategy, Bank Advertising New, 21(8/9), 6.

Reichheld, F. \& Sasser, W. (1990). Zero defections: Quality comes to service, Harvard Business Review, 68(5), 105-111.

Richins, M. (1997). Measuring emotions in the consumption experience, Journal of Consumer Research, 24(2), 127-146.

Ruyter, K. d., J, B., \& Peeters, P. (1997). Merging service quality and service satisfaction An empirical test of an integrative model, Journal of Economic Psychology, 18(4), 387496. 
Sanchez, J., \& Moliner, M. A. (2006). Customer perceived value in banking services, International Journal of Bank Marketing, 24(5), 266-283.

Sanchez, J., Callarisa, L., Rodríguez, R. M., \& Moliner, M. A. (2006). Perceived value of the purchase of turism product, Tourist Management, 27, 394-409.

Sheth, J., Newman, B., \& Gross, B. (1991). Why we buy what we buy: A theory of consumption values, Journal of Business Research, 22(2), 150-170.

Smith, J. B., \& Colgate, M. (2007). Customer value creation: a practical framework, Journal of Marketing Theory \& Practice, 15(1), 7.

Sørensen, J. (2008). Measuring emotions in a consumer decision-making contextapproaching or avoiding, Working Paper Series Department of Business Studies Aalborg University, 20, 2-43.

Sweeney, J. S. (2001). Consumer perceived value: the development of multiple item, Journal of Retailing, 77(2), 203-220.

Teehan, R., \& Tucker, W. (2010). A simplified lean method to capture customer voice, International Journal of Quality and Service Science, 2(2), 175-188.

Valenzuela, L., Garcia de la Madariega, J., \& Blasco, M. (2007). Orientación al valor del cliente y las nuevas métricas de marketing. Revisión y análisis, Panorama Socioeconómico, 25, 70-75.

Womack, J., \& Jones, D. (1996). Lean thinking. New York: Free Press.

Womack, J., \& Jones, D. (2005). Lean consumption, Harvard Business Review, 83(3), 58-68.

Womack, J., \& Jones, D. (2005). Lean solutions: How companies and customers can create value and wealth together. London, U.K.: Simon \& Schuster.

Woodruff, R. (1997). Customer value: The next source for competitive edge, Journal of the Academy of Marketing Scienc, 25(2), 139-153.

Zeithaml, V. (1988). Consumer perceptions of price, quality, and value: A means-end model and synthesis of evidence, Journal of Marketing, 52(3), 2-22. 